\title{
$-0$ \\ On Socio-Economic Roles and Specialization
}

\author{
Robert P. Gilles' \\ Emiliya Lazarova² \\ Pieter H.M. Ruys ${ }^{2}$
}

' Virginia Tech, Blacksburg, USA, and Tinbergen Institute, The Netherlands;

2 Tilburg University, The Netherlands. 


\section{Tinbergen Institute}

The Tinbergen Institute is the institute for economic research of the Erasmus Universiteit Rotterdam, Universiteit van Amsterdam, and Vrije Universiteit Amsterdam.

Tinbergen Institute Amsterdam

Roetersstraat 31

1018 WB Amsterdam

The Netherlands

Tel.: $\quad+31(0) 205513500$

Fax: $\quad+31(0) 205513555$

Tinbergen Institute Rotterdam

Burg. Oudlaan 50

3062 PA Rotterdam

The Netherlands

Tel.: $\quad+31(0) 104088900$

Fax: $\quad+31(0) 104089031$

Please send questions and/or remarks of nonscientific nature to driessen@tinbergen.nl.

Most TI discussion papers can be downloaded at http://www.tinbergen.nl. 


\title{
On socio-economic roles and specialization*
}

\author{
Robert P. Gilles \\ Department of Economics, \\ Virginia Tech, Blacksburg, USA \\ Emiliya Lazarova \\ Department of Econometrics, \\ Tilburg University, the Netherlands \\ Pieter H.M. Ruys \\ Department of Econometrics, \\ Tilburg University, the Netherlands
}

March 2006

\begin{abstract}
:
Xiaokai Yang's theory of economic specialization under increasing returns to scale is a formal development of the fundamental Smith-Young theorem on the extent of the market and the social division of labor. In this theory specialization-and, thus, the social division of labor-is firmly embedded within a system of perfectly competitive markets. This leaves unresolved whether and how such development processes are possible in economies based on more primitive, non-market organizations.

In this paper we discuss a general relational model of economic interaction. Within this non-market environment we discuss the emergence of economic specialization and eventually of economic trade and a social division of labor. We base our approach on three levels in organizational development: the presence of a stable relational structure; the presence of relational trust and subjective specialization; and, finally, the emergence of objective specialization through the institution and the social recognition of economic roles.
\end{abstract}

\footnotetext{
* This paper is dedicated to the memory of Xiakai Yang, whose work on specialization and the social division of labor inspired us in constructing the theory as presented and discussed in this paper. This paper is an extended compilation of the formal theory developed in Gilles, Lazarova and Ruys (2006).

C Corresponding author: Department of Economics, 3016 Pamplin Hall, Virginia Tech, Blacksburg, VA 24061, USA. Email: rgilles@vt.edu.
} 


\section{Introduction}

Xiaokai Yang visited the Center for Economic Research at Tilburg University during the spring of 1999. Immediately he engaged two of the three authors in extensive discussions on his research program. We easily identified similar research interests and this led to some fruitful exchanges and discussions.

During Professor Yang's visit to Tilburg we in particular discussed working paper versions of papers that were published subsequently as Diamantaras, Gilles and Ruys (2003), and Sun, Yang, and Zhou (2004). These papers address some of the central problems and theoretical questions that lie at the intersection of our respective research programs. It is therefore fitting that in this paper we return to these central questions and sketch a new theory of the emergence of a social division of labor in a non-market economy.

The research program of Professor Yang was seminally developed in Yang (1988) and subsequently brought to fruition in numerous research papers ${ }^{1}$. The core of this research program is the application of inframarginal analysis to the decision model of an individual consumer-producer within a system of perfectly competitive markets. Yang's inframarginal approach is in turn used to model the Smith-Young approach to the relationship of specialization, the social division of labor, and increasing returns to scale, in line with Smith (1776), Young (1928), and Stigler (1951).

Smith (1776) argued in his seminal work The Wealth of Nations that the social division of labor is limited by the extent of the market, so that the benefits of specialization to an individual are determined largely by the existing social division of labor in the economy. This is also known as the Smithian Theorem. Young (1928) extended the Smithian Theorem into a synergetic argument that the extent of the market also depends on the level of social division of labor. Thus, the presence of increasing returns leads to specialization and further social division of labor. In turn, a high level of social division of labor within a system of competitive markets leads to the deepening of increasing returns to scale and eventually to increasing economies of specialization. These developments form further incentives to specialize and to develop the social division of labor.

In the present paper we intend to sketch an argument that extends the Smithian theorem beyond the setting of a economy based on a system of perfectly competitive markets. Our argument is that the Smith-Young mechanism also applies to social organizations and institutional settings other than that of a system of perfectly competitive markets. In particular, we develop a theory based on value generating binary relationships - or "matchings" - that describe the relational foundation of all economic interaction. This describes a primitive economy in which markets at best exist in their most primitive form, namely as a network of binary exchange relations; thus, without necessarily the presence of a single price that determines the terms of trade in marketable exchange relationships.

We conclude from our analysis that the process of specialization occurs at different levels of embeddedness of the individual consumer-producer within the relational structure of the economy. Only at its most advanced state - namely that of objective specialization - this process results into a social division of labor. A social division of labor thus generates

\footnotetext{
${ }^{1}$ We refer to Yang (2001), Yang (2003), and Cheng and Yang (2004) for a comprehensive review of the work that has been accomplished in this research program.
} 
economic development and growth in the context of more primitive economic institutions and exchange mechanisms. This leads to the conclusion that a social division of labor can emerge independently from a system of perfectly competitive markets. The Smith-Young mechanism is in fact founded on more fundamental forces within the economy. Hence, economic development is not based on the endogenous selection of a specialization by an individual based on the prevailing market prices; instead, it is the institutionalization of a pair of complementary social economic roles - from which the individual selects one - that generates growth. Each role corresponds to some objectively defined specialization of that individual. For a detailed development of the mathematical model we refer to Gilles, Lazarova and Ruys (2006) on which the current paper is based.

In their seminal contribution, Yang and Borland (1991) already have shown theoretically that the Smith-Young mechanism functions as a determining factor for economic growth. Indeed, the mechanism of ever-deepening economic specialization and the accompanying development of the social division of labor lead to significant growth. In economic history and the new institutional economics this has been accepted as the main engine behind the rise of the western economies. ${ }^{2}$

Recently, Acemoglu et al. (2005) have confirmed Yang and Borland's theoretical conjecture through empirical observations and analysis. Furthermore, Ogilvie (2004) and Greif (2006) have extended this institutional growth argument and pointed to economic organizations other than the perfectly competitive market in which the Smith-Young mechanism causes economic development and growth. Acemoglu et al. (2005) mainly point to the development of property rights and the underlying political institutions as causes of economic growth, while Ogilvie (2004) investigates the development of medieval and earlymodern guild systems. Empirical evidence of past performance of western economies backs up these arguments. Our theoretical contribution fits with this line of research.

In Section 2 we develop our model of a matching economy based on binary valuegenerating activities among economic agents. We also discuss subjective specialization and the possibility of subjective stability through means of an existence result. In Section 3 we define generic stability as our main equilibrium notion and present our main existence result. This result identifies the possibility of objective specialization, which in turn implies the emergence of a social division of labor in such a matching economy. We summarize and extend our main line of thought in Section 4.

\section{Trust and stability}

As mentioned above, in the current paper we discuss a model of a rather primitive economy in which economic agents directly interact with one other without reference to a central organization such as a system of competitive markets or a unified price mechanism. Instead individual economic agents engage in binary, value-generating relationships or "matchings". These matchings have to be understood as binary productive engagements, which are not necessarily trade relationships. It is assumed in this very primitive economy that every individual activates exactly one value-generating matching.

\footnotetext{
${ }^{2}$ See, e.g., North and Thomas (1973), North (1990), Greif (1994), and North (2005).
} 
Our main argument is that there are two different forms of stability possible within such a matching economy.

- Subjective stability: Individuals engage in binary, value-generating relationships-socalled matchings-and stability is attained if individuals are not willing to either break all of their engagements and become autarkic or switch partners for higher benefits. Thus, there results a stable matching pattern based on the local properties of these binary engagements. In other words, the presence of stability is "subjective" in the sense that it is completely based on the properties of the productive abilities and utility functions of the individuals in the economy.

We show that the presence of stable matching patterns is guaranteed in case a special substructure of potential relationships has a bipartite structure. The existence of subjecttively stable matching patterns is the subject of our first existence theorem.

If a state of subjective stability is attained, individuals might develop mutually beneficial trade within the relationship that they are engaged in. Subsequently, after beneficial trade has been established, the engaged individuals might specialize their productive activities. This specialization occurs only within the (subjective) setting of the matching that they are engaged in. We call this subjective specialization.

We emphasize that subjective specialization does not induce a social division of labor since individuals are not engaged at a higher social plane; their economic interaction is explicitly limited to be confined to their matchings only. In that regard the organization of the economy remains scattered and there is no unified social organization of the economy. As a consequence, there are no widespread gains from trade. There are only locally generated gains from trade.

- Generic stability: Only if generic stability is possible, economic agents can truly specialize in an objective fashion and there emerges a social division of labor. A matching economy attains generic stability if for every profile of utility functions and production sets, there exists a stable matching pattern. Our main existence theorem states that such generic stability is attained if and only if there is a social organization of the economy based on at least two socially recognized economic roles. Hence, there should exist at least two complementary socio-economic roles such that value-generating relationships solely exist between individuals with different socio-economic roles. Only after such complementary socio-economic roles are established, a true endogenous social division of labor can emerge in which individuals specialize in these roles. In turn, this implies that a social organization of the economy emerges and that widespread gains from trade become attainable.

Our main existence theorem on generic stability thus identifies that a bipartite social division of labor is a pre-requisite for stability. This amends the Smithian theorem in the sense that there has to exist a finite set of socio-economic roles into which individuals can specialize, to establish stability in the social organization of the economy. The emergence of a set of socially recognized socio-economic roles is, thus, a necessary condition for true generic stability in the economy.

Since economic prosperity is determined largely by the set of available complementary socio-economic roles, the Smith-Young mechanism of economic development is now linked to the development of this set of socially recognized roles; innovation in social 
organization-in the sense that new social roles are developed-now determines the extent of the market and, thus, economic growth.

Although our model of a matching economy describes a very primitive society, we believe that it makes possible some rather deep conclusions. Our approach also makes explicit again the indeterminacy problem identified by Gilles and Diamantaras (2005). They argued that the theory of the Smith-Young development mechanism within the context of a well-developed system of perfectly competitive markets is founded on a circular argument: prices of traded goods determine individuals' specialization and, thus, prices determine the social division of labor. This, in turn, determines which goods are produced and traded, determining the extent of the market. This brings up the question who or what ultimately determines which goods are traded and how economic development is accomplished. In other words, this development mechanism has no origin or starting point. Gilles and Diamantaras concluded that the determinacy problem has to be resolved in order to make further progress on the analysis of economic development processes. Our goal in our analysis is to show that stability is required for any specialization of individuals in an economy and that a social division of labor only emerges in a society that has a stable organizational structure based on objectively given socio-economic roles. In this section we discuss the first part of this argument. We introduce a very generic model of a society in which individuals can engage into binary economic interactions. We define two stability concepts in such a relational economy and determine when stability is attainable.

In our approach we put in some sense this determinacy problem at the center of our analysis. Indeed, our main result states that generic stability requires the existence of a certain set of established social roles from which individuals can choose when they specialize. Each socio-economic role stands for a certain commonly recognized economic specialization and in equilibrium the number of agents of each role is balanced. ${ }^{3}$ Only then an effective social division of labor emerges and the society can engage into an effective process of economic development and growth. Ultimately, economic development is thus founded on the enhancement and extension of the socially determined set of accepted economic roles.

We conclude that economic development and growth is caused by organizational and institutional change rather than technical change only. ${ }^{4} \mathrm{We}$ argue that technical change is a consequence and expression of the effectiveness of the social organization of the economy.

\subsection{Matching economies and stable matching patterns}

Formally, we denote by $N=\{1, \ldots, n\}$ a finite set of individuals. At this stage we do not make any assumptions about these individuals regarding their individual abilities. Hence, at this stage we do not explicitly assume that these individuals are even able to specialize in any form. Instead we endow these individuals with the abilities to engage into relational economic activities that generate economic values or wealth. ${ }^{5}$ We refer to these binary interactions as

\footnotetext{
${ }^{3}$ For the latter argument we refer to Yang's theory of general equilibrium under endogenous specialization. Explicitly we refer to Yang (2001) for a detailed discussion and treatment of this argument.

${ }^{4}$ We refer again to Acemoglu et al. (2005) for a complete discussion of this point of view.

5 The most primitive form of a matching is that of cooperation in some production activities. More advanced forms include the simple exchange or trade of two commodities. The gains from trade then form the values that are generated between the two traders.
} 
value-generating (economic) relational activities. Thus, individuals are assumed to have relational economic abilities. We do not exclude that these relational abilities in turn might be based on individual abilities. We do not assume or impose that these value-generating relational activities take place in the context of a market. Instead we assume that these relational abilities describe the economy itself.

Formally, we define $\Gamma \subset\{i j \mid i, j \in N\}$ as a set of potential relational activities between the individuals in $N$. Here, for two distinct individuals $i \in N$ and $j \in N$ with $i \neq j$, we define $i j \in \Gamma$ to mean that these individuals $i$ and $j$ are able to engage in a value-generating relational activity. We indicate this potential relational engagement $i j \in \Gamma$ as a potential matching of $i$ and $j$.

Furthermore, every individual $i \in N$ is endowed with complete and transitive preferences over her potential matchings $L_{i}(\Gamma)=\{i j \in \Gamma \mid j \in N\} \subset \Gamma$ in which she can engage. These preferences can be represented by a hedonic utility function given by $u_{i}: L_{i}(\Gamma) \rightarrow \Re$. Let $u=\left(u_{1}, \ldots, u_{n}\right)$ denote a hedonic utility profile and $U$ be the set of all hedonic profiles representing complete and transitive preferences. We summarize the developed primitive concepts into a unifying concept:

\section{Definition 1}

A matching economy is defined to be a triple $\mathrm{E}=(N, \Gamma, u)$ in which $N$ is a finite set of individuals, $\Gamma$ is a potential matching structure on $N$, and $u \in U \dot{U}$ is a hedonic utility profile on $\Gamma$. The pair $(N, \Gamma)$ consisting of the set of individuals endowed with all potential valuegenerating relationships is also called the (relational) constitution of $\mathrm{E}=(N, \Gamma, u)$.

A matching economy is essentially based on potential binary activities that generate economic values. For example, a trade economy can be represented as a matching economy between buyers and sellers who can trade physical goods to generate gains from trade. Hence $\Gamma$ represents the binary trade relations between such buyers and sellers. The gains from trade are exactly the hedonic utilities generated in these trade relationships, i.e., $u_{i}(i j)$ stands for the individual gains from trade of individual $i$ as she engages in a mutually beneficial trade with individual $j$.

Within the context of a matching economy we investigate the proper definition of stability. Stability refers to the presence of a pattern of activated matchings that is in a state of equilibrium. Stability is a necessary condition for the further development of an economy, in particular for the emergence of specialization and a social division of labor.

Our main hypothesis in the definition of stability is that in a matching economy $\mathrm{E}=(N, \Gamma, u)$ each individual $i$ activates exactly one of her potential matchings in $L_{i}(\Gamma)$. This fundamental hypothesis is founded on the fact that we model a very primitive economy without the presence of advanced economic or social institutions. In such a primitive economy it is natural to assume that individuals only interact with a single other individual at a time and that more complex interactions require more advanced social institutions than assumed within our context. 
Formally we introduce the notion of a matching pattern to describe a collection of activated binary value-generating relationships in $\Gamma$.

\section{Definition 2}

A matching pattern is a subset of the potential matching structure $\pi \subset \Gamma$ such that every individual is either paired with exactly one other individual or remains relationally autarkic, i.e., $\pi \subset \Gamma$ is such that either $\left|L_{i}(\pi)\right|=1$ or $i i \in \pi$, for all $i \in N$.

In a matching pattern one and only one matching is selected and executed by each individual. For ease of notation we denote the utility an individual $i$ has when participating in a matching pattern $\pi$ in which $L_{i}(\pi)=\left\{i i_{\pi}\right\}$ as $u_{i}(\pi)$, i.e., $u_{i}(\pi) \equiv u_{i}\left(i i_{\pi}\right)$, for all $i \in N$. We emphasize that the hedonic utility profile considered here allows an individual to consider only one matching at a time, since we do not allow an individual to engage in multiple matchings at the same time.

With the tools developed so far we are able to introduce two relational stability concepts. Again we let the matching economy $\mathrm{E}=(N, \Gamma, u)$ be given throughout. For matching pattern $\pi \in \Pi$, a potential matching $i j \in \Gamma \backslash \pi$ is a blocking matching if $u_{i}(i j)>u_{i}(\pi)$ as well as $u_{j}(i j)>u_{j}(\pi)$.

Having defined a blocking matching as a strict binary Pareto improvement, we follow the concepts used in the literature on matching (Roth and Sotomayor, 1990). We point out that our notion of stability is closely related to that of stability in network formation (Jackson and Wolinsky, 1996). With this concept we can define our stability property of a matching pattern.

\section{Definition 3}

A matching pattern $\pi \in \Pi$ is stable in the economy $\mathrm{E}=(N, \Gamma, u)$ if all matchings in $\pi$ satisfy the individual rationality (IR) and the no blocking (NB) conditions:

- IR $u_{i}(\pi) \geq u_{i}($ ii) for all $i \in N$, and

- $\quad N B$ there is no blocking matching with regard to $\pi$, that is, for all $i \in N$ and $j \in N$ with $i \neq j$ and $i j \in \Gamma \backslash \pi$ it holds that $u_{i}(i j)>u_{i}(\pi)$ implies that $u_{j}(i j) \leq u_{j}(\pi)$.

Stable matching patterns in $E$ are denoted by $\pi \in \Pi *(N, \Gamma, u)$.

Condition (IR) is an individual rationality requirement, that states that an individual cannot be matched with another individual without her consent, i.e., if an individual is better-off under relational autarky, she will pursue that.

Condition (NB) stands for a non-blocking condition requiring that a blocking matching does not exist with respect to matching pattern $\pi \in \Pi$. Under (NB) if an individual prefers to be matched with an alternative individual than the one with whom he is matched under matching pattern $\pi$, then that alternative individual does not agree to engage with him. This condition is closely related to the condition of link addition proofness in network formation. Link addition proofness is at the foundation of the notion of pairwise stability in network formation, seminally introduced by Jackson and Wolinsky (1996). 
With reference to the definition of stable matching patterns, we note that in general these stable patterns might fail to exist. In particular, in the absence of such a stable matching pattern there emerges a state of permanent chaos or instability within a relational economy E. This essentially refers to a situation in which individuals permanently search for the most optimal partner to engage in a value-generating relationship.

The main application of the general relational framework developed is here that of a relational economy of consumer-producers. We point to the new classical framework developed in Yang (2001) and Yang (2003). The new classical approach is firmly founded on the premise that consumer-producers specialize within a social context of a structure of (market) interactions and, thus, attain higher welfare levels.

In Gilles et al. (2006) we start at an even more primitive level of reasoning. Before there is actual specialization, there are consumer-producers with simple skills on which these specializations can be based. We recognize that skills, unlike commodities, are intrinsic to a consumer-producer and cannot be exchanged. They can, however, be shared. Sharing one's skills with another individual is a process that does not make the giver any poorer in the skill. ${ }^{6}$ As established by Yang and Borland (1991) and Yang (2003), learning-by-doing is an important mechanism in the process of growth. However, in Yang's framework this process is individual-specific, i.e., economic individuals are not allowed to learn from each other. In Gilles et al. (2006), we go beyond this restriction by allowing learning processes among engaged individuals.

\subsection{Existence of stability and subjective specialization}

In our previous discussion, we have focused mainly on a primitive economy with limited specialization. In such economies there might not emerge an equilibrium in the form of a stable matching pattern. (For an explicit example of such an economy we refer to Example 3.4 in Gilles et al. (2006).) Here we investigate the sufficient conditions for the existence of such stable matching patterns. We also discuss the implications of our findings with regard to specialization in a relational economy.

We first address the introduction of a particular sub-structure of the constitution of a matching economy.

\section{Definition 4}

A matching pattern $\pi \in \Pi$ is weakly stable in the economy $\mathrm{E}=(N, \Gamma, u)$ if all matchings in $\pi$ satisfy the individual rationality (IR) and whenever a blocking matching $i j \in \Gamma \backslash \pi$ exists, at least one of the blocking partners in ij is relationally autarkic, i.e., $\{i i, j j\} \cap \pi \neq \varnothing$.

Denote by $\Pi_{w} \subset \Pi$ the collection of all weakly stable matching patterns.

Finally, we define $\Gamma_{w}=\cup \Pi_{w} \subset \Gamma$ to be the set of all potential weakly stable matchings.

\footnotetext{
${ }^{6}$ A commodity, in comparison, if shared makes the giver poorer in the possession of that commodity. This is to say that while commodities are pure private goods, skills are non-rival in nature.
} 
The definition introduces a sub-constitution $\left(N, \Gamma_{w}\right)$ based on the class of weakly stable matching patterns. We refer to the sub-constitution $\left(N, \Gamma_{w}\right)$ as the core-constitution of the matching economy $\mathrm{E}=(N, \Gamma, u)$.

For the formulation of our existence theorem we introduce some auxiliary concepts. Let $\Omega \subset \Gamma$ be some collection of mutually beneficial relationships between individuals in $N$. The sub-constitution $(N, \Omega)$ is bipartite if there exists a partitioning $\left\{N_{1}, N_{2}\right\}$ of the set of individuals $N$ such that

$$
\Omega \subset\left\{i j \mid i \in N_{1} \text { and } j \in N_{2}\right\} \cup\{i i \mid i \in N\} \text {. }
$$

Hence, in a bipartite constitution of a relational economy, there are two socially recognized economic roles or "types" such that value-generating relationships only exist between individuals of different types.

\section{Existence theorem 1}

If a matching economy $\mathrm{E}=(N, \Gamma, u)$ has a bipartite core-constitution $\left(N, \Gamma_{w}\right)$, then it holds that $\Pi *(N, \Gamma, u) \neq \varnothing$.

The existence theorem stated above identifies a particular set of potential value-generating relationships that has to form a bipartite structure in order for stable matching patterns to be possible. Hence, certain vital or "constituting" relationships have to form a bipartite structure. This implies that there are essentially two groups of individuals between which these constituting relationships exist. We again refer to Gilles et al. (2006) for further discussion of this topic and its consequences.

\section{Specialization and stability}

In the previous section we have introduced various notions of stability - or equilibrium - and linked the existence of stable matching patterns to the bipartite nature of certain vital valuegenerating relationships. In this section we link the existence of these stable matching patterns with the possibility of individual specialization.

\subsection{The emergence of exchange and subjective specialization}

Now assume that we have a matching economy E that satisfies the condition of Existence Theorem 1, i.e., the economy $\mathrm{E}$ has a bipartite core-constitution. In that case there emerges a state of subjective stability in the sense that there exists a stable matching pattern in the economy. We emphasize that subjective stability is fully based on the local conditions in the economy, i.e., the explicit abilities of the individuals in the economy rather than the generic, global conditions in the economy.

If a subjectively stable state emerges in the economy, all individuals are engaged in stable value-generating relationships. Within the context of these relationships, a moderate 
level of relational trust can be build up. If a sufficient high level of trust emerges, there first will come about commodity exchange between these individuals. Thus, the emergence of a level of moderate relational trust allows individuals to engage in mutually beneficial exchange of commodities. We emphasize that this commodity exchange is purely binary and, thus, scattered at best. Therefore, there is neither any market nor any global trade network emerging.

After mutually beneficial commodity exchange has been established to enhance the value-generating relationships in which individuals are engaged, a further deepening of the level of relational trust will take place. After the relational trust has been enhanced, individuals will be trustworthy enough of their partner that they become willing to specialize their productive activities. We emphasize that this form of specialization is completely based on the local conditions in the economy, in particular the conditions in the relationship in which individuals are engaged. Indeed, if conditions are favorable, individuals might develop sufficient trust towards their partner such that they decide to specialize fully and exchange their necessary commodities with their like-wise fully specialized partner. This can be indicated as subjective specialization.

Gilles et al. (2006) develop a detailed example in which these specialization processes are made explicit and higher levels of wealth or hedonic utility are achieved through specialization and binary exchange. Again we emphasize that under subjective specialization there does not emerge a global social organization in the form of a system of markets and a social division of labor. Instead individuals remain engaged in binary relationships guided by conditions in their immediate neighborhood, i.e., by local conditions in the economy only.

\subsection{The emergence of trade and objective specialization}

The previous discussion clarifies the emergence of stable matching patterns and of subjective specialization. This emergence is essentially based on localized conditions based on features within the subjectively stable pattern of stable matchings. For an economy to have persistent access to such gains from specialization, the social structure of the economy has to generically admit stable matchings. Hence, whatever capabilities and desires of the individuals-represented by their utility functions and (possibly) other individualistic features-a stable matching pattern has to exist in the matching economy.

Technically, this brings up the question under which conditions on $(N, \Gamma)$ there exists a stable matching pattern for every possible matching economy $\mathrm{E}=(N, \Gamma, u)$, where $u$ is an arbitrary utility profile. This line of research follows the research agenda set in the matching literature. Here we are able to apply the main result of Papai (2004).

\section{Definition 5}

A relational constitution $(N, \Gamma)$ is generically stable if for every utility profile $u \in U$-and thus for every matching economy $\mathrm{E}=(N, \Gamma, u)$ based on this relational constitution-it holds that $\Pi *(N, \Gamma, u) \neq \varnothing$.

Our main existence theorem can now be stated as follows: 


\section{Existence theorem 2}

The relational constitution $(N, \Gamma)$ is generically stable if and only if $(N, \Gamma)$ is bipartite.

This second existence theorem provides a complete and powerful characterization of generically stable matching structures. This is a very strong result with some deep consequences. As stated before, certain sets of skill complementarities might result into the emergence of stable matching patterns. These stable matching patterns in turn give rise to subjective specialization and mutually beneficial exchange. This does not mean that there results widespread gains from trade. For such enhanced economic development it is necessary that there emerges an objective or socially recognized division of labor.

In particular, the deepening of the stable matching patterns through subjective specialization in turn leads to the emergence of bipartite structures of potential matchings. This emergence is based on the social recognition of the roles that are based on the subjective specialization of individuals in such stable matching patterns. This is discussed next.

In objective specialization each individual now expects to be trading when she engages in a matching. Also, under objective specialization, unlike under subjective specialization, the level of trust expands to the whole set of players, i.e., to the whole economy. This is why an individual believes fully that she can be matched with another player with whom exchange is beneficial in a stable matching. In fact, there is common knowledge that individuals with different socially recognized specializations can be matched in highly productive value-generating relationships. Individuals, who assume social roles, have socially justified beliefs that a stable matching pattern exists.

At present we argue that further deepening of the efficiency in this economy is only possible through the establishment of a true social division of labor based on a set of socially recognized economic roles. For that purpose we consider a primitive society in which individuals acquire two different productive skills, hunting skills and gathering skills. Assume that at first there emerges some stable matching pattern in which individuals exploit the complementarities of hunting and gathering. Within the context of this equilibrium, individuals now decide to become slightly specialized in hunting or gathering. If sufficient trust is developed among matched individuals, some individuals might specialize fully on hunting or gathering. This introduces subjective specialization into this primitive economy similar to the social developments discussed at the end of section 3.1.

Subsequently, a deepening of this specialization might emerge. Indeed, if these subjective specializations are recognized socially, individuals become a "hunter" or a "gatherer". Being a hunter now becomes a socially recognized economic role, as does being a gatherer. This in turn implies that only after the establishment of such complementary social roles there emerges a social division of labor. ${ }^{7}$ The emergence of socially recognized economic roles and the accompanying social division of labor is now identified with the effects of full-blown economic specialization. This type of specialization is generic in the sense of Definition 5 above; it can be referred to as objective specialization.

It should be noted here that the emergence of trade between individuals with different social roles is fundamentally different from commodity exchange between subjectively

\footnotetext{
${ }^{7}$ We emphasize here that the establishment of a social role requires the social recognition of each role and the separation of the related specialism from each individual. Thus, the social recognition of an economic role induces a dichotomy of this role and other aspects of her life for every individual that assumes this role.
} 
specialized individuals. Social recognition indeed alleviates the informational burden and implements certain expectations.

Finally, we can in fact identify conditions under which a competitive market can emerge. If sufficiently large number of individuals assume the social roles of hunter and gatherer and other economic institutions such as the protection of property rights, monetary instruments, and the creation of actual market places are established, then there might emerge a market at which hunters and gatherers can trade vegetables and meat for a well established and unique market price.

Objective specialization excludes relationships between individuals with the same social role as being potentially beneficial economic matchings. This implicitly reduces the potential matching structure to an odd acyclic or bipartite structure in which only matchings between individuals with two different roles are recognized.

\section{From chaos to generic stability and market systems}

In this paper we introduced a four-stage approach to the emergence of a social division of labor based on the objective specialization of individuals. As a fifth stage we can add the emergence of market institutions themselves. This approach clarifies that the presence of a social division of labor is in fact a prerequisite for the creation or emergence of a functioning price mechanism. Summarizing these four stages are:

Stage I: Chaos (non-equilibrium). In a primitive relational economy without objective specialization, there usually are conditions that do not support an equilibrium. This leads to a situation in which all individuals are fully autarkic and in which there is a state of permanent relational chaos. Individuals are fully self-reliant for the provision of necessities for survival. Consequently, the generated level of welfare is at the level of pure subsistence. Any additional utility generated through interpersonal spillovers from social interaction are purely additional benefits to the generically low subsistence levels.

Stage II: Primitive equilibrium. Within a primitive relational economy there might exist conditions that allow the emergence of a stable social interaction pattern. Such a stable pattern is only founded on subjective and personal features, not on any objective or social conditions. Within this stage we distinguish two sub-stages.

(II-A) At first there only emerges a stable pattern in which interpersonal spillovers are exploited. This first level of stable social interaction facilitates the emergence of a moderate level of subjective trust among the matched individuals.

(II-B) Next, the emergence of sufficient subjective trust among the individuals that are engaged with each other, supports the introduction of exchange among those individuals; the exploitation of interpersonal spillovers is extended into the exchange of economic commodities leading to even higher levels of utilities. The emergence of exchange is an important step into the development of an economy. 
Stage III: Subjective specialization. After exchange has been established there is the possibility for a further deepening of interpersonal trust within the stable relationships in the economy. This facilitates the emergence of subjective specialization in which individuals based on the demands of their interpersonal relationships specialize their economic activities. Hence, within the context of a stable exchange relationship with another individual, an individual selects a production plan to optimize his utility level based on the resulting consumption plan.

This process of subjective specialization is similar to the specialization process based on inframarginal analysis developed by Yang - as a formalization of the Smith-Young development mechanism - within the context of a perfectly competitive price mechanism. However, subjective specialization does not take place within the context of a functioning price mechanism, but rather within the interpersonal relational setting of each individual separately.

Stage IV: Objective specialization. The emergence of subjectively specialized individuals can lead to the recognition of socio-economic roles in the society at large. Hence, individuals specializing subjectively on certain skills within the context of their individual value-generating relationships, become socially recognized as occupying a "profession" that relates to this specific skill-set. Thus, professionals are identified in the society as occupying a certain socio-economic role. This corresponds to an objectification of the specialization of that individual: the individual assumes in fact an objectively defined and socially recognized economic role.

Subsequently, there emerge social rules related to these socio-economic roles. Returning to our illustrative example of a hunter-gatherer society, the engagement of a socially recognized "hunter" with a socially recognized "gatherer" in an economically beneficial (exchange) relationship thus becomes the foundation for economic development. Individuals subsequently specialize in an objective fashion; they now select from a given set of socio-economic roles and engage in an objective fashion with other individuals in their respective social roles to generate mutual economic benefits.

It is only within this context of objective specialization that there emerges a social division of labor which further development acts as an engine for economic growth described in the context of a market by the Smith-Young mechanism.

Stage V: Market emergence. We argue that only after the establishment of a social division of labor based on the social recognition of certain economic roles, there can emerge a functioning market or price mechanism. Besides the social division of labor there have to be established other economic institutions. Only after these other conditions are met, there might emerge a price mechanism through which further economic growth and development is made possible in the form of the Smith-Young mechanism based on the extent of the market.

In this paper we only have developed the most basic principles of a descriptive theory. The main conclusion is that economic development and growth are closely related to the objective development of an extended set of socio-economic roles in a society. These social roles have an objectively, public nature and as such should be subject to a purely public economic 
theoretical analysis or an evolutionary treatment. This is closely related to the conclusion of Gilles and Diamantaras (2005).

Further development of this abstract theory of matching economies is required before we can expect a full and working understanding of the five-stage process of market development summarized above. This is left to future research.

\section{References}

Acemoglu, D., S. Johnson and J. Robinson (2005), "Institutions as the Fundamental Cause of Long-Run Growth", in Handbook of Economic Growth, edited by P. Aghion and S. Durlauf, Elsevier North-Holland, Amsterdam.

Cheng, W., and X.K. Yang (2004), "Inframarginal Analysis of Division of Labor: A Survey", Journal of Economic Behavior and Organization, 55, 137-174.

Diamantaras, D., R.P. Gilles, and P.H.M. Ruys (2003), "Optimal Design of Trade Institutions", Review of Economic Design, 8, 269-292.

Gilles, R.P., and D. Diamantaras (2005), "New Classical Economics: Towards a new paradigm in economics?", Division of Labour and Transaction Costs, 1, 35-56.

Gilles, R.P., E. Lazarova and P.H.M. Ruys (2006), "Stability, Specialization and Social Recognition", CentER Discussion Paper 2006-17, Tilburg University, Tilburg, the Netherlands.

Greif, A. (1994), "Cultural Beliefs and Organization of Society: A Historical and Theoretical Reflection on Collectivist and Individualist Societies", Journal of Political Economy", Journal of Political Economy, 102, 912-950.

Greif, A. (2006), Institutions and the Path to the Modern Economy: Lessons from Medieval Trade, Cambridge University Press, Cambridge, UK.

Jackson, M.O., and A. Wolinsky (1996), "A Strategic Model of Social and Economic Networks", Journal of Economic Theory, 71, 44-74.

North, D., and R. Thomas (1973), The Rise of the Western World: A New Economic History, Cambridge University Press, Cambridge, UK.

North, D. (1990), Institutions, Institutional Change and Economic Performance, Cambridge University Press, Cambridge, UK.

North, D. (2005), Understanding the Process of Economic Change, Princeton University Press, Princeton, NJ 
Ogilvie, S. (2004), "Guilds, Efficiency, and Social Capital: Evidence from German ProtoIndustry”, Economic History Review, 57, 286-333.

Papai, S. (2004), "Unique Stability in Simple Coalition Formation Games", Games and Economic Behavior, 48, 337-354.

Roth, A.E., and M. Sotomayor (1990), Two-Sided Matching: A Study in Game-Theoretic Modeling and Analysis, Cambridge University Press, Cambridge, UK.

Smith, A. (1776), An Inquiry into the Nature and Causes of the Wealth of Nations, Reprint: University of Chicago Press, Chicago, IL.

Stigler, G. (1951), "The Division of Labor is Limited by the Extent of the Market", Journal of Political Economy, 59, 185-193.

Sun, G., X.K. Yang and L. Zhou (2004), "General Equilibria in Large Economies with Endogenous Structure of Division of Labor", Journal of Economic Behavior and Organization, 55, 237-256.

Yang, X.K. (1988), A Microeconomic Approach to Modeling the Division of Labor Based on Increasing Returns to Specialization, $\mathrm{PhD}$ thesis, Princeton University, Princeton, NJ.

Yang, X.K. (2001), Economics: New Classical Versus Neoclassical Frameworks, Blackwell Publishing, Malden, MA.

Yang, X.K. (2003), Economic Development and the Division of Labor, Blackwell Publishing, Malden, MA.

Yang, X.K., and J. Borland (1991), "A Microeconomic Mechanism for Economic Growth", Journal of Political Economy, 99, 460-482.

Young, A. (1928), "Increasing Returns and Economic Progress", Economic Journal, 38, 527-542. 